\title{
STUDIES ON THE STRUCTURE AND FUNCTIONS OF THE PAPILLARY RIDGES OF THE DIGITAL SKIN IN LEPROSY
}

\author{
By A. Paul Jayaraj, F R.m.s. \\ Central Food Technological Research Institute, Mysore \\ and \\ *D. S. Chaudhury, m.B.B.S. \\ Gandhi Memorial Leprosy Foundation, T. Narisipur, Mysore
}

It is generally assumed that there are different sensory modalities associated with the specific sensory receptors of skin. Nerve endings of the fibrillary structures are found in hairy and non-hairy skin in relation to certain cells of the basal cell layer of the epidermis where the nerve endings and the cell constitute as sensory receptors. Touch sensibility of hairy and hairless skin are of different orders. In hairy skin it is arranged into a spot pattern and in non-hairy skin it is evenly spread over the surface papillary ridges.

The histological structure of the leprosy lesion in the skin is very distinctive. In lepromatous leprosy, the epidermis is thinned. The cell layers are flattened and slightly irregular. The papillary ridges are completely flattened and compressed. There is a free subepidermal zone where cellular infiltrations are characteristically absent. In tuberculoid leprosy, the epidermis is thinned in the particular area where the lesion starts. In advanced tuberculoid leprosy, the papillary ridges are completely flattened and irregular. The sub-epidermal zone in contrast to lepromatous leprosy, does not appear as a clear zone but is invaded by the inflammatory infiltrate reaching up to the epidermis. This clearly shows that in leprosy irrespective of type the structural damage to the epidermis is more. The purpose of this investigation is to study the changes that occur to the papillary ridges in leprosy and to determine the relation of those changes to alterations of cutaneous sensibility found in leprosy. Digital skins were primarily chosen for the investigation as the touch sensibility in this part of the skin is evenly spread over the papillary ridges in normal individuals.

\section{Materials and Methods}

Thirty pieces of skin were taken from the distal pads of the fingers from thirty patients showing typical lesions of leprosy. 20 were taken from lepromatous leprosy and 10 from tuberculoid

*Present address. :

Medical Officer (Leprosy)

Ministry of Health,

Accra, Ghana, West Africa. 
cases. There were no visible lesions anywhere on the finger pads. Biopsies were also taken from lesions of each patient to confirm the type of lesion histologically. Tissues were fixed in 10\% neutral formalin and frozen sections were taken at 20 micron thickness and stained by the method described by Balasubramanyam, JayaraJ and Gass (1954) for nerve fibres. 20 sections were taken from each specimen for this study. Remaining tissues were processed for paraffin sections. Sections were stained with haematoxyline and eosin and for acid-fast bacilli by the method described by JAYARAJ (1955). 5 specimens were taken from the distal pad of fingers of normal individuals as control.

\section{Observations on Cutaneous Sensibility}

Tactile sensibility was observed with the tip of a fine feather on the distal pad of the fingers before biopsies were taken. Stimuli were applied with lighter touch to heavier touch and with fine pin points.

It was found that in early lepromatous leprosy the finger pads responded to light touch and in advanced lepromatous leprosy the site was anaesthetic to light touch but responded to heavy touch. Occasionally the finger pads were found to be anaesthetic to heavy touch but responded to fine pin pricks.

In tuberculoid leprosy the finger pads did not respond to heavy touch with the feather but responded to pin pricks. Occasionally they were anaesthetic to pin pricks also.

\section{Results}

Changes in the epithelial pattern in relation to nerve endings in papillary ridges in lepromatous leprosy: The epithelium is flattened leaving the surface of the papillary ridges in limited length in the corium. The ridges are prominently seen even though the epithelium is flattened. The ridge is embedded in loose connective tissue and its deep surface is in contact with the ascending nerve fibre of the corium. The ascending fibre is seen damaged by way of ballooning. There are numerous neural fragments seen embedded in the papillary ridges. The sweat passages are seen in the middle of some of the papillary ridges. There is no marked difference in histology between the early lesion and advanced lesion excepting that the papillary ridges are found to vary in length. The ridges project more in length in the corium in early lepromatous leprosy and less in advanced lepromatous leprosy.

\section{Changes in Tuberculoid Leprosy}

The thickness of the epithelium is reduced. In some places, the epithelium is flattened and there are no papillary ridges seen. The subepithelial part of the corium shows no nerve fibres. Very 


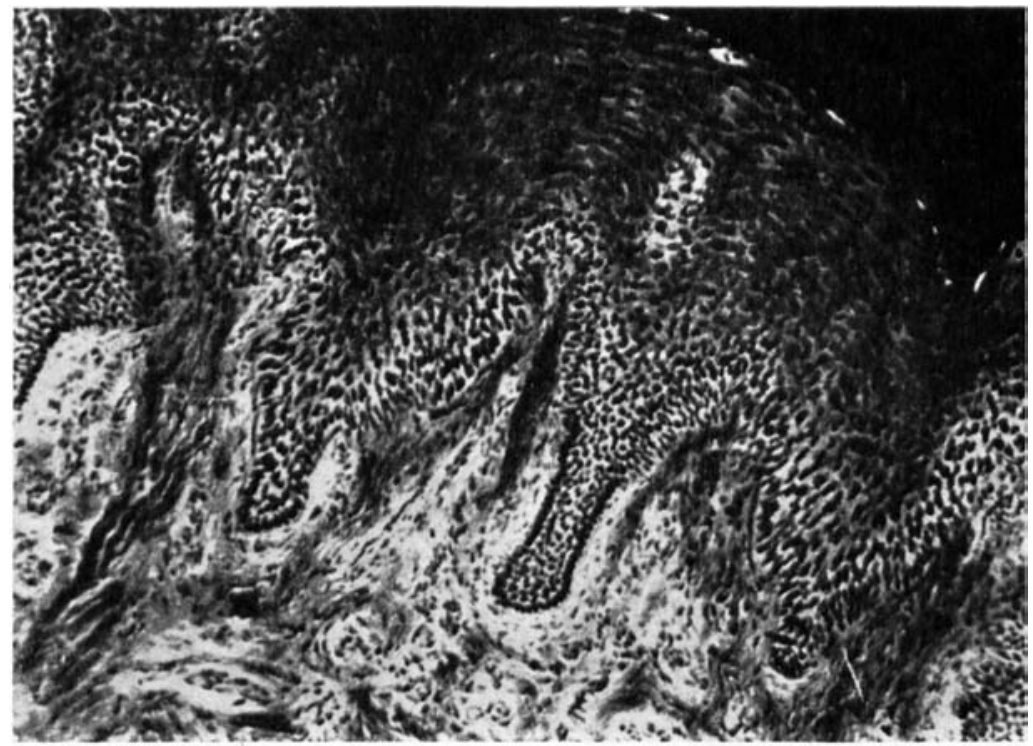

FIci. 1. Transverse section of the digital skin showing the papillary ridges and the sub-epidermal nerve plexus underneath the ridges. Distal pad of the middle finger, Male, 30 years-Manual worker x 100.

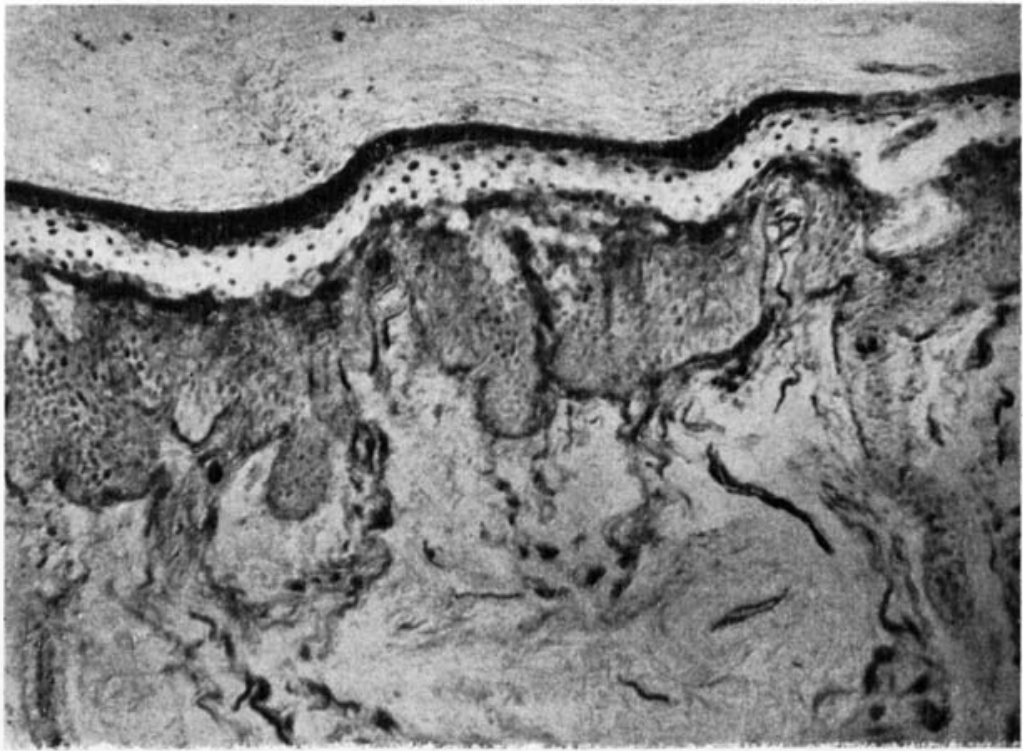

FIG. 2. Transverse section of the digital skin showing flattened epithelium with limited papillary ridges descending less deeply into the corium and the subepidermal nerve plexus underneath the ridges. Distal pad of the ring finger, male, 25 years. Lepromatous $\times 100$. 


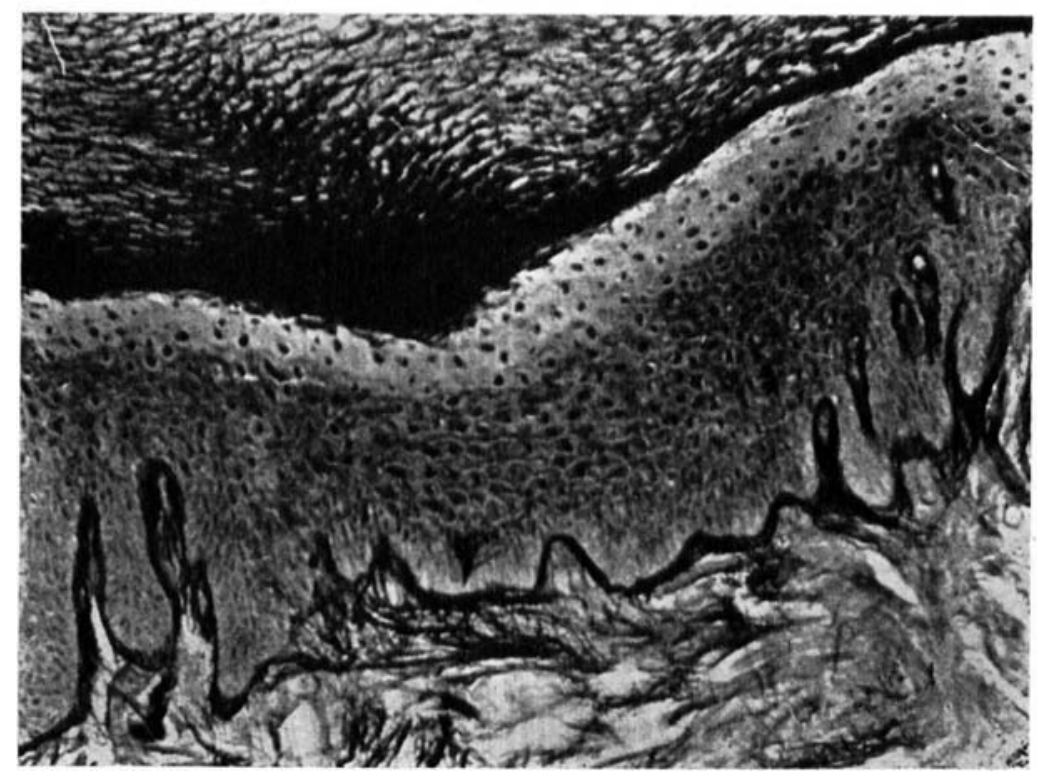

Fici. 3. Transverse section of the digital skin showing the epithelium with flattened papillary ridges and the sub-epidermal area completely invaded by connective tissue elements. Distal pad of the middle finger, male, 27 years, Tuberculoid x 100 .

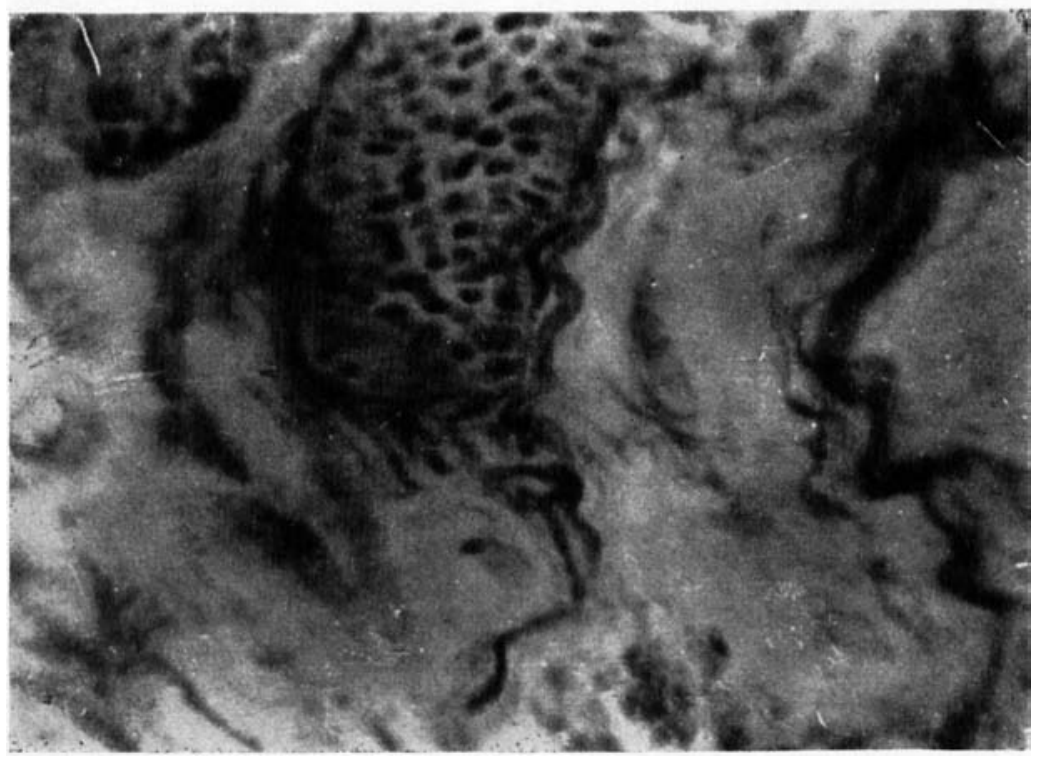

FIG. 4. Transverse section of the digital skin showing the papillary ridge in contact with extensive nerve plexus. Distal pad of the ring finger, male, 25 years. Lepromatous x 400 . 


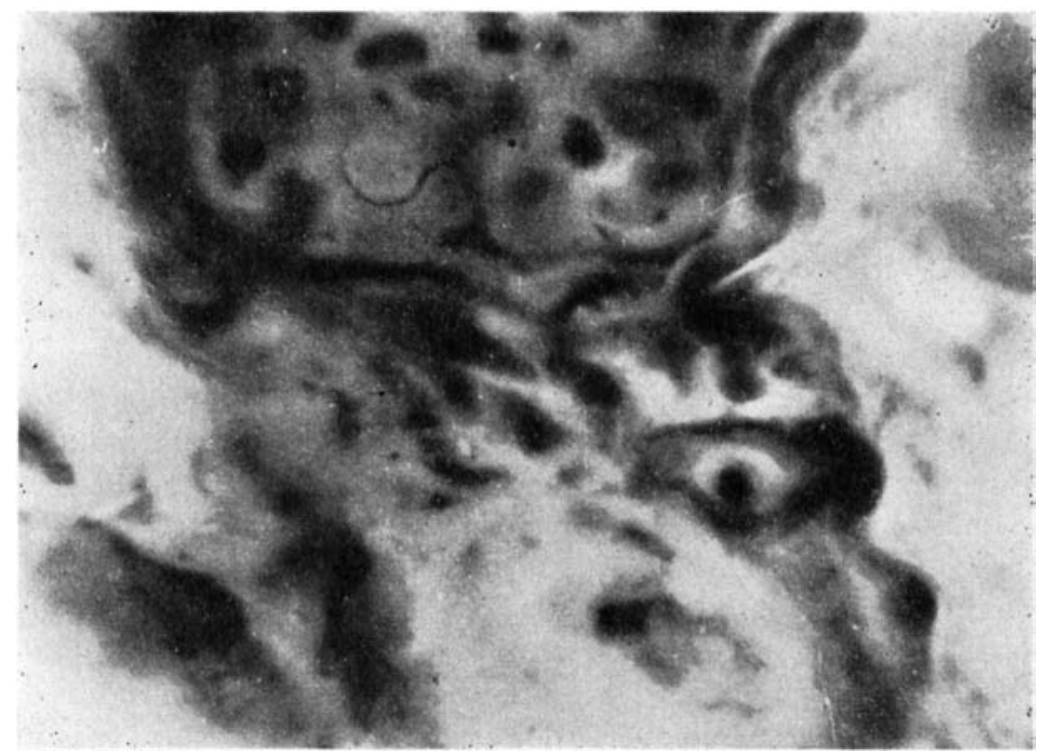

FIG. 5. Higher magnification of fig: 4. showing the epidermal nerve ending on the papillary ridge $\mathrm{x} 900$.

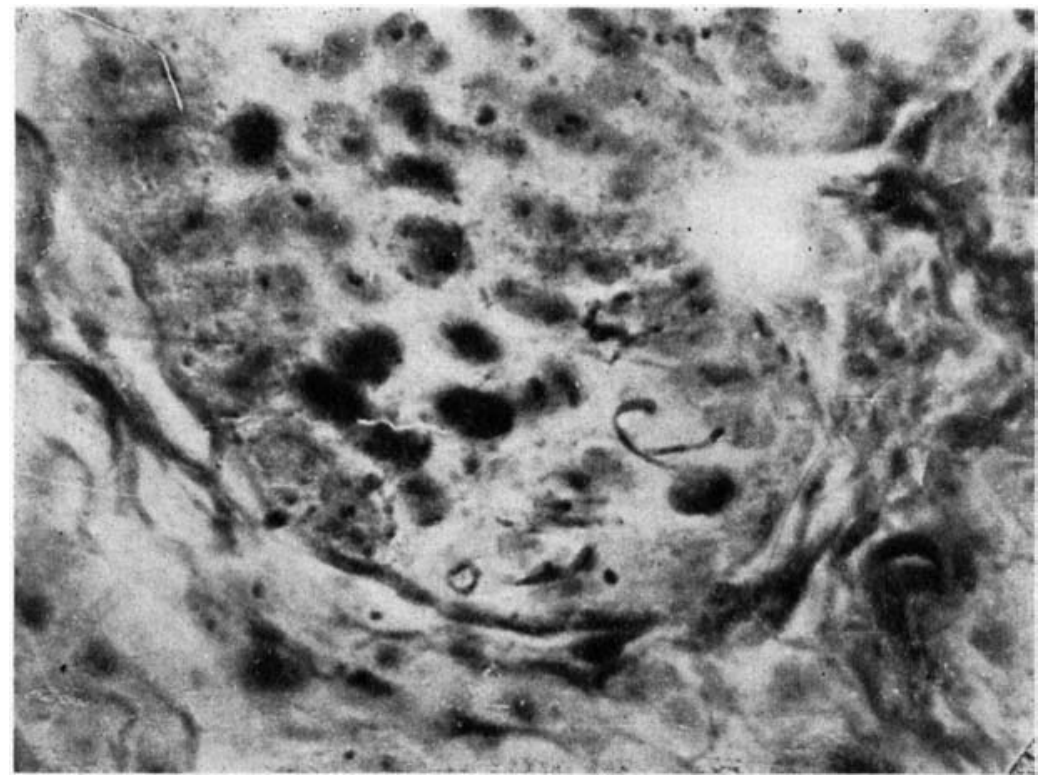

Fig. 6. Transverse section of the digital skin showing the neural fragments situated in the papillary ridge. Distal pad of the middle finger, male, 25 years. Lepromatous $\mathrm{x} 900$. 
occasionally fine nerve fibres are seen coursing towards the epithelium. Nerve endings in relation to any part of the epithelium is absent. However there are some fine nerve fibres seen coursing towards the Meissner corpuscle.

\section{Discussion}

The stratum corneum of the digital skin is much thicker than that of the hairy skin. Yet the finger tip performs the function of an efficient tactile sense organ with high sensibility and discrimination. CAUNA (1954) observed that the papillary ridges contain separate receptors for tactile acuity and discrimination, and the Meissner corpuscle is a selective receptor for pressure that coincides with the axis of the corpuscle and is primarily concerned with tactile discrimination. He has beautifully demonstrated a rubber model of the papillary ridges showing the function of the intermediate ridge and the Meissner corpuscle. The intermediate ridge is embedded in loose connective tissue and is not attached to the corium. It is in contact with an extensive nerve plexus with epidermal nerve endings. CAUNA has shown that the intermediate ridge follows the movements of the papillary ridge acting as a magnifying lever mechanism for transmission of touch stimuli to the underlying receptors. It can be equally stimulated through different surface areas of the papillary ridge and is primarily concerned with tactile acuity. Even though the epithelium is flattened in lepromatous leprosy the intermediate papillary ridges in the digital skin are seen distinctly and the ridges are definitely in contact with extensive sub-epidermal nerve plexus.

These findings on the basis of histological and functional considerations justify that the papillary ridges in lepromatous leprosy continue to act as part of essential tactile sense organ. JAYARAJ and Chaudhury (1961) have shown that the Meissner corpuscles in lepromatous leprosy look almost normal even though the bacilli are situated alongside the neuro-fibrillary ramifications. The present investigation further confirms that the sensory modalities are not much altered even though the nerve bundles are invaded by bacilli and cellular infiltration. In tuberculoid leprosy, the papillary ridges are practically non-existent and further the sut-epidermal nerve plexus is destroyed resulting in severely impaired sensory function.

\section{Summary}

1. Biopsies from distal pad of the fingers from 30 leprosy patients comprising 20 lepromatous and 10 tuberculoid were studied by cytological nerve staining methods.

2. It was found that the papillary ridges of the digital skin in lepromatous leprosy are very distinct. In early lepromatous leprosy, the intermediate ridges descend more in length into the corium 
compared to advanced lepromatous leprosy where the ridges descend less in length. Extensive nerve fibres are seen embedded in these papillary ridges.

3. In tuberculoid leprosy, the intermediate papillary ridges do not descend into the corium and the ascending nerve fibres are mostly destroyed, practically resulting in severe sensory impairment.

4. The histological and functional considerations justify that the papillary ridges in lepromatous leprosy continue to act as an essential part of the tactile sense organ.

\section{Acknowledgments}

Our grateful thanks are due to Dr. V. Subrahmanyan, D.SC., F.R.I.C., F.N.I., Director, Central Food Technological Research Institute, for kindly offering all facilities and encouragements to this investigation and to Dr. R. V. Wardekar, B.SC., M.D., Secretary, Gandhi Memorial Leprosy Foundation, for kind permission to do this investigation. We are also indebted to Dr. M. Swaminathan, D.SC., F.N.I., Assistant Director, Central Food Technological Research Institute, Mysore, for his kind and valued encouragements. We express our gratitude to Mr. K. V. Achyuta Rao, for his aid with the photographic work.

\section{References}

1. Balasubramanyan, M. Jayaraj, A. P. and Gass, H. H. (1954), "An Improved Histological Method for the examination of cutaneous nerve in leprosy". Leprosy Review, 15, 83-86.

2. JayaraJ, A. P. (1955) "Periodic Acid in the staining of Acid-fast Bacilli in Tissue Section", J of the Anat. Soc. of India Vol. 4, 41-42.

3. CaUnA, N. (1954) "Nature and Functions of the Papillary Ridges of the Digital skin", The Anatomical Record Vol. 119, 449-458.

4. Jayaraj, A. P. and Chaudhury, D. S. (1961) studies on the Neuro-Histological changes in the Meissner Corpuscle in Leprosy-Leprosy Review, Vol. 32, $153-157$. 